Revista Signos

2008, 41(67)

$257-278$

\title{
Leer y escribir en una escuela chilena: Representaciones discursivas de los diferentes agentes educativos en las áreas prioritarias del currículo escolar en NB3
}

\author{
Alejandra Meneses* \\ Universidad Diego Portales \\ Chile
}

Resumen: En este artículo se describen e interpretan -mediante la construcción de categorías lingüísticas y discursivas- las representaciones discursivas sobre leer y escribir de los diferentes agentes escolares en las áreas prioritarias del currículo escolar en el nivel educativo NB3. El corpus -constituido por discursos auténticos (planificaciones, clases, textos escolares y evaluaciones) y entrevistas- ha sido obtenido mediante un acercamiento etnográfico interpretativo a la realidad educativa. El análisis del discurso se basa en el modelo dialógico de tres entradas (Peytard \& Moirand, 1992; Moirand, 2006) lo que permite construir las representaciones utilizando unidades no tópicas que articulan el análisis del corpus construido heterogéneamente desde el punto de vista de los géneros discursivos. Se concluye, que al interior de la cultura escolar conviven dos tipos de representaciones: a) leer y escribir para manejar y producir información propia de una visión de la educación como desarrollo de competencias; b) leer y escribir en cuanto representación de la escuela tradicional: leer literatura como patrimonio ilustrado y la escritura ligada a la conservación de la lengua: ortografía, vocabulario y caligrafía. Asimismo, se observa que al interior del aula la lectura y la escritura se utilizan, principalmente, como un instrumento de reproducción de información: copiado y acceso de la información (dialogismo intertextual). Dichas representaciones son interpretadas dentro del sistema educativo como parte de los efectos retroactivos sobre los procesos de enseñanza-aprendizaje de las pruebas estandarizadas que se aplican, actualmente, en los establecimientos educacionales chilenos para medir la calidad educacional (dialogismo interaccional).

Palabras Clave: Representaciones discursivas, interdiscursividad, dialogismo, lectura y escritura, agentes educativos.

Recibido: 30-VIII-2007

Aceptado: 11-XII-2007
*Becaria Proyecto MECESUP UCV-0004, Doctorado en Lingüística PUCV.

Correspondencia: Alejandra Meneses (alejandra.meneses@udp.cl). Tel.: (56-02) 6762390. Fax: 6762502. Facultad de Educación, Universidad Diego Portales. Vergara 210, Santiago, Chile. 


\title{
Literacy events in a Chilean school: Discursive representations of different scholar agents in the main areas of the scholar curriculum in the NB3 level
}

\begin{abstract}
This paper describes and interprets -through the construction of linguistic and discursive categories- the discursive representations about literacy events of the different scholar agents in the main areas of the scholar curriculum in the NB3 level. The corpus, built from real discourses (plannings, lessons, textsbooks and evaluations) and interviews, was obtained through an interpretative ethnographic approach to the educational reality. The discourse analysis is based on three inputs of the dialogical model (Peytard and Moirand, 1992; Moirand 2006). This allows the building of the discursive representations using non-topic units that articulate the discourse analysis built heterogeneously from the points of view of the discursive genres. It is concluded that inside the school culture two representations live together: a) literacy events to manage and produce information, characteristic of a vision of education as the development of competencies; $b$ ) the representation of literacy events from the traditional school: reading literature as an illustrate patrimony and writing as a conservation of language: orthography, vocabulary and calligraphy. At the same time, it is observed that in the lessons, literacy events are used, mainly, as a mechanism to reproduce information: copying and accessing information (inter-textual dialogism). These representations inside the school system are interpreted to be part of the retroactive effects on the educational processes of the standard tests being currently applied in the Chilean schools to measure quality of education (interaction dialogism).
\end{abstract}

Key Words: Discursive representations, interdiscursivity, dialogism, literacies events, scholar agents.

\section{INTRODUCCIÓN}

La escuela se ha caracterizado por ser el lugar del aprendizaje formal de la lectura y la escritura como medio para acceder al conocimiento. Es el lugar en el que el estudiante entra en contacto permanente con la cultura escrita y con textos referidos a las diferentes áreas del conocimiento. La elección de esta problemática responde a la urgencia de acercarse, desde la complejidad propia del proceso educativo, a la forma en que los agentes de la cultura escolar chilena expresan, en sus discursos auténticos y cotidianos, su concepto de lectura y de escritura en cuanto fenómeno que atraviesa la mayoría de los quehaceres del ser humano moderno. Esta investigación busca, en consecuencia, describir en profundidad, desde una perspectiva sociocultural y mediante el análisis lingüístico-discursivo, las distintas maneras como se representan los distintos agentes educativos, al interior de una escuela chilena, la lectura y la escritura. No hay, hasta el momento, estudios detallados que aborden cómo se construyen, al interior de las acciones pedagógicas, los roles de lector y escritor en distintas disciplinas escolares, ni cómo se conciben las actividades de leer y escribir en ciencias, sociedad o en matemática, ni qué tipos de textos se producen y se leen al interior de aula, ni los modos de leer y escribir a través de las diversas áreas curriculares del sistema educativo chileno. 
Ahora bien, es fundamental situar este estudio interpretativo en su contexto: la seria crisis por la que atraviesa el sistema educativo chileno. Desde los años 90 , se ha dado un proceso constante de reforma del sistema escolar, cuyo objetivo ha sido el mejoramiento de la calidad y la equidad de la educación. Para ello, se han llevado a cabo cambios curriculares y una creciente inversión en infraestructura, materiales curriculares y remuneraciones de los docentes. Sin embargo, como lo muestran pruebas estandarizadas de evaluación, tanto nacionales como internacionales, no ha habido aumentos significativos en la calidad de los aprendizajes y estos se distribuyen desigualmente por tipos de establecimiento y condiciones socioeconómicas de los estudiantes (MINEDUC, 2004a, 2004b). Es más, precisamente, las prácticas pedagógicas al interior de la escuela -núcleo del proceso educativo- no han sido intervenidas ni consideradas como objeto de estudio.

Por otra parte, estamos inmersos en una época altamente tecnologizada en la que las redes de información nos proveen un número incalculable de mensajes. Sin embargo, la razón última de este fenómeno no se encuentra en el espectacular desarrollo de las tecnologías de comunicación, sino más bien en el hecho que la sociedad valora como un recurso para su propia organización la transmisión de información (Morandé, 2003). Entonces, cabe preguntarse cómo se concibe la lectura y la escritura en el sistema educativo desde este nuevo escenario y cómo se vincula con la construcción del conocimiento escolar en sociedades funcionales y complejas. Ello porque, en la llamada sociedad de la información, el ser humano -censurando la pregunta por el porqué- acepta la retroalimentación de las propias operaciones cognitivas y admite naturalísticamente la fragmentación del saber. En la actualidad, el ser humano es capaz de acceder a la información deseable en cualquier campo del conocimiento, del funcionamiento de la sociedad y de la estructura del propio ser humano; sin embargo, ¿se puede llegar, realmente, a afirmar que sabe más de sí mismo? (Morandé, 2003).

\section{Marco de referencia}

\subsection{Las representaciones discursivas: el acercamiento desde el dialogismo}

La teoría de las representaciones discursiva nos permite explorar las acciones pedagógicas desde el análisis de los discursos que configuran esta esfera social para identificar y contrastar las funciones que cumplen la lectura y la escritura en las diversas áreas del conocimiento escolar al interior de la comunidad escolar.

Adam (2005) define la representación discursiva o esquematización -situándola en el marco del análisis del discurso y la lingüística textual- como todo acto de referencia en cuanto construcción operada desde y por el discurso de un locutor y como (re)construcción por parte de un intérprete. Es, en este sentido, la expresión de un punto de vista sobre un asunto dado que va 
asociado a los propósitos comunicativos de toda interacción. Lo anterior lleva a concluir que la construcción de sentido no es una relación transparente y unívoca con respecto a los referentes acerca de los que se quieren comunicar, sino que, precisamente, dicha selección y la puesta en enunciación son inseparables de los propósitos comunicativos (Adam, 2005).

La esquematización/representación discursiva es, por tanto, una noción que articula tanto lo textual con lo discursivo como con el anclaje psicosocial del proceso de intercambio comunicativo. Asimismo, incorpora la dimensión de proceso a la actividad discursiva, ya que tanto la noción de discurso como la de texto -como señala Adam $(1999,2005)$ - se refieren más bien al resultado de una práctica discursiva. La representación discursiva se vincula tanto con los aspectos discursivos como con los textuales de la lengua en uso y mantiene relaciones ascendente y descendente con el orden del discurso y con el orden textual respectivamente, lo que permite para el análisis la determinación de categorías discursivas y lingüísticas para su análisis.

La representación discursiva no es nunca la de un individuo; en efecto, desde la lógica natural -campo desde el cual se gestó esta noción- Grize (1996) formula como postulado básico para la interacción comunicativa el dialogismo bajtiniano. Por lo tanto, la construcción de una representación verbal como puesta en enunciación depende, evidentemente, de sus representaciones sociales, de sus preconstruidos culturales, lo que no quiere decir que exista una relación de condicionamiento, sino más bien de condición, por considerar: el ser humano, al estar situado, está siempre inserto en un contexto histórico-social, pero, sigue manteniendo la posibilidad del cambio y la transformación social. En definitiva, sigue siendo libre.

Sin embargo, si se pretende distinguir categorías descriptivas para el análisis de datos empíricos, no se advierte con claridad cómo operar con dicho principio, Moirand (2001, 2003b, 2005, 2006) propone para el análisis índices lingüísticos que, al ser localizados en la superficie textual, permiten la rigurosidad del mismo. A su vez, dichos índices son interpretados poniéndolos en relación con lo social y lo histórico. Se pueden determinar dos tipos de relaciones que configuran este dialogismo: relaciones interdiscursivas definidas por las relaciones que todo enunciado tiene con otros enunciados sobre un mismo objeto y relaciones interlocutivas definidas como las relaciones que todo enunciado tiene con las respuestas anticipadas de los destinatarios presentes o virtuales (Moirand, 2005). De esta manera, el dialogismo -según se refiera al objeto o a los interlocutores- va a ser distinguido como intertextual e interaccional (Moirand, 1999, 2001, 2005) en estrecha relación con las dos dimensiones que configuran el discurso: representacional y comunicativa. El dialogismo intertextual se refiere a aquel hilo de hechos y de otros discursos identificados y recopilados a lo largo del intradiscurso (Moirand, 1999) mediante el estudio del paradigma designación/denominación y la composición de los textos. Por su parte, el dialogismo interaccional se refiere a las posibles intervenciones que el enunciador anticipa en el texto en relación con los destinatarios y el sobredestinatario presentes en todo discurso. Indicios de este 
dialogismo interaccional son marcas de persona, formas de exhortación, preguntas retóricas (Peytard \& Moirand, 1992).

En definitiva, el principio de dialogismo bajtiniano y la operacionalización de Moirand (2005) permiten articular la compleja relación entre lengua, comunicación y contextualización histórica y social en cuanto memoria y tradición de otros discursos. En otras palabras, se asume que todo discurso está en una relación dinámica con otros discursos y, por ende, es inseparable de una memoria interdiscursiva. "El discurso se encuentra con el discurso del otro en todos los caminos que conducen a su objeto, y no puede no entrar con él en una interacción viva e intensa" (Charaudeau \& Maingueneau, 2005: 170). Asimismo, permite acercarse al discurso desde dos ejes: el horizontal, en el cual se van engarzando una a otras las diferentes unidades que componen el texto (principio de composicionalidad), y el vertical, que emerge en el eje horizontal donde aparecen huellas de esos otros discursos (principio de dialogismo) (Moirand, 2004a, 2005).

Con respecto a la relevancia del estudio de las representaciones en el campo educativo, Cambra (2003) destaca que los profesores gestionan y planifican sus clases según las imágenes que poseen sobre la realidad educativa y su función. Por lo mismo, es novedoso y necesario ampliar la cuestión de las representaciones a los diferentes agentes educativos y en las áreas prioritarias del currículo escolar, pues la escuela en cuanto cultura escolar está compuesta por esta diversidad de agentes que se comunican y que ponen en común sus imágenes sobre la educación, el tipo de ser humano que se quiere formar, las metodologías educativas, entre otras. Por lo tanto, la lectura y la escritura como actividades escolares permiten acercarse a las representaciones de lo que es considerado primordial para la escuela de hoy, en cuanto están insertas en el sistema educativo chileno y en el marco más amplio de la sociedad de la información. Precisamente, interesa dar cuenta tanto de la homogeneidad como de la heterogeneidad de voces que configuran las representaciones de la lectura y la escritura; más aún, es fundamental, precisamente, hallar las fracturas y desajustes entre dichas voces. De ahí, la relevancia del "principio de dialogismo" para acercarse a este objeto de estudio.

En síntesis, las representaciones discursivas, en cuanto imágenes localizables en los discursos permiten, por una parte, levantar interpretaciones sobre las concepciones subyacentes de lo que es leer y escribir al interior de una comunidad escolar y, al mismo tiempo, construir dicha representación a partir de la actuación pedagógica del profesor materializada a través de discursos auténticos que concretizan su quehacer: planificaciones, interacciones didácticas, evaluaciones que permiten observar el discurso del hacer (lo proyectado, lo realizado, lo evaluado) en relación con la lectura y la escritura. Por otra parte, es importante contrastar dicho discurso con el discurso del decir sobre el hacer con el fin de hallar fracturas relevantes para una posterior intervención pedagógica. Cabe destacar que al considerar la cultura escolar como un espacio comunicativo complejo y constituido por diferentes agentes, es necesario considerar 
también los discursos de los otros agentes educativos: administración pública (primer nivel de concreción curricular), proyecto educativo y curricular del establecimiento educacional (segundo nivel de concreción curricular) y familia.

\section{Marco metodológico}

Partiendo del supuesto ontológico de que la realidad social no está construida exclusivamente por el lenguaje, sino que este es un medio de comunicación a través del cual se generan, circulan y se transforman representaciones y relaciones sociales, se define el discurso desde un paradigma interpretativo como instrumento para la creación de significados compartidos por grupos sociales y se delimita la designación del concepto a la escuela de análisis del discurso de tendencia francesa (Peytard \& Moirand, 1992; Maingueneau, 2003, 2005; Charaudeau \& Maingueneau, 2005; Narvaja, 2006). El análisis se realiza a partir de un corpus de datos heterogéneos obtenido del acercamiento etnográfico a una cultura escolar concreta.

La elección de un acercamiento etnográfico a la realidad educativa se justifica, por una parte, por la necesidad de generar datos contextualizados sobre la escuela como lugar social de aprendizaje formal y, por otra, en la urgencia de restituir a los propios agentes del sistema educativo la voz y el protagonismo que les corresponde en la relación educativa; de ahí, entonces, el interés de estudiar la realidad educativa desde el punto de vista de sus propios agentes (punto de vista émico). Por otra parte, el acercamiento etnográfico permite la comprensión e interpretación de los discursos, ya que aporta significación relevante sobre el contexto de producción in situ, el que, generalmente, es reconstruido a partir del texto o a través de la recolección de datos dispersos no sistematizados. El ingreso del investigador al campo por un período prolongado de tiempo (cinco meses) y la convivencia con los agentes de la cultura escolar, permiten afirmar que entre método etnográfico y análisis del discurso se puede acceder a la relación desentrañable entre texto y contexto; sobre todo, ayuda a acercarse al contexto más allá del microcontexto (Moirand, 2003b), puesto que los discursos son definidos como tejidos de intertextualidades en los que se pueden localizar indicios que permiten reconstruir las representaciones en cuanto preconstruidos y co-construidos de la temática en estudio.

En cuanto a la elección del análisis del discurso de tendencia francesa como método y técnica de análisis de los datos, es fundamental precisar que dicho enfoque articula la relación entre lo lingüístico, lo discursivo y lo social, no solo trabajando con lo que se dice (dictum), sino también con el cómo se dice (modus); cuestión fundamental para acercarse a la dimensión explícita/implícita presente en toda construcción discursiva.

Si bien el método etnográfico ha permitido la recolección de la información; para responder a la problemática en estudio, la construcción del corpus responde a decisiones de reorganización de 
la información, la que está en estrecha relación con criterios analíticos provenientes del análisis del discurso. Para ello se seguirá la propuesta de Moirand (2004b): corpus de referencia y corpus de trabajo. En primer lugar, es preciso determinar que la construcción de este corresponde a la recolección de discursos localizados (géneros de una misma institución recolectadas mediante la etnografía) (Peytard \& Moirand, 1992). En términos generales, se trata de un corpus transversal (Peytard \& Moirand, 1996; Charaudeau, 2000), cuya característica principal es la heterogeneidad discursiva (planificaciones de los docentes, libros de textos escolares, interacciones áulicas, entrevistas, programas de estudio), además de toda la información recolectada mediante la etnografía que forma parte de la cultura escolar (reglamento de convivencia, conversaciones informales, cuadernos de los estudiantes, entre otros). El criterio establecido para la construcción de dicho corpus es la exhaustividad y la vinculación con la temática en estudio.

No obstante, se ha construido un corpus de trabajo (Moirand, 2004a, 2004b) en consonancia con el objetivo general de esta investigación. Esto quiere decir que, partiendo de la evidencia de la imposibilidad de cierre de todo corpus de discursos auténticos (Moirand, 2004b), se delimita este mediante la noción operatoria de momento discursivo (Charaudeau \& Maingueneau, 2005) -utilizada en los estudios de discursos de divulgación científica en medios de comunicación. Dicha noción ha sido resignificada, en este estudio, a través del uso de la unidad de temporalización didáctica que permite delimitar y organizar la información de manera contextualizada. Dos criterios se han escogido para la construcción del corpus de trabajo: grupo curso (análisis centrado en las interacciones realizadas por el quinto básico A correspondiente al nivel educativo NB3) y unidad didáctica (discursos que configuran el proceso educativo completo mediante dicha unidad de organización educativa.)

Una vez definido el corpus de trabajo, se determinó la configuración de dos subcorpus en relación con los objetivos de la investigación y con la configuración del sistema de enseñanza-aprendizaje, con el propósito de dar sistematicidad y distinguir focos articulatorios que permitieran la construcción de las representaciones discursivas.

El análisis del discurso se basa en el modelo dialógico de tres entradas (Peytard \& Moirand, 1992; Moirand, 2006), que considera todo discurso en cuanto configurado por dos dimensiones: comunicativa y cognitiva (Peytard \& Moirand, 1992; Moirand, 2003a). Dicho modelo permite abordar la cuestión de las representaciones discursivas desde la localización en el intradiscurso de huellas interdiscursivas (intertextuales e interaccionales) que permiten poner en relación un conjunto de discursos heterogéneos que circulan dentro de una cultura en cuanto complejo espacio comunicativo. Para operar con dicho modelo es preciso distinguir categorías lingüísticas y discursivas que faciliten la observación y análisis de los discursos recogidos. En términos generales, en la dimensión cognitiva se estudiaron las operaciones y huellas de la designación/ denominación y la reformulación de nociones y la composicionalidad de los discursos en relación 
son las operaciones cognitivo-discursivas (Moirand, 2003b), pues todo discurso, al comunicar, representa objetos, agentes y procesos de un mundo real o ficticio. Por otra parte, es fundamental considerar que al construir un universo discurso, la representación de dicho universo en el discurso se construye en relación con el modo en que se evalúa la situación de comunicación y en el tipo de vinculación que se establece con un destinatario y sobredestinatario: dimensión comunicativa. El estudio de las ubicaciones o lugares enunciativos (marco comunicativo: preanálisis y operaciones enunciativas) y la vinculación con los discursos de otros en términos tanto de dialogismo interaccional como intertextual antes mencionados, forman parte de esta dimensión comunicativa del discurso.

Partiendo de la pregunta directriz de esta investigación - ¿cómo se representan los agentes educativos de una cultura escolar, en sus discursos, la lectura y la escritura en las áreas prioritarias del currículo escolar, específicamente, en el ciclo educativo NB3?- se ha realizado el análisis en torno a dos subcorpus: el subcorpus 1 centrado en el quehacer del profesor en cuanto responsable del tercer nivel de concreción del currículo escolar. Este subcorpus compuesto por los discursos que materializan las acciones e interacciones pedagógicas del docente ha sido abordado desde cuatro dimensiones de análisis: lo proyectado, lo realizado, lo evaluado y lo declarado. En consecuencia, se ha construido centrándose en la actuación didáctica del docente (Cambra, 2003), pues es el responsable último de la enseñanza-aprendizaje y es quien entra en interacción permanente con los estudiantes. Se busca, pues, en este subcorpus identificar todas las huellas y marcas de las representaciones de la lectura y la escritura en relación con las actividades de enseñanza y aprendizaje. De lo que resulta que el subcorpus 1 está compuesto tanto por los discursos que materializan las acciones pedagógicas que realiza el docente fuera de la sala de clase (discursos previos) como por las interacciones didácticas dentro de la sala de clase (discursos co-construidos e imprevistos).

En el subcorpus 2, configurado desde los discursos de los otros agentes educativos (programas de estudio, entrevistas a directivos y entrevistas a los estudiantes), se han analizado las tensiones y claves interpretativas que llevan a comprender las representaciones discursivas que circulan al interior de la cultura escolar. En cuanto a este subcorpus, está compuesto por los discursos de los otros agentes que configuran el sistema de enseñanza-aprendizaje y que influyen directa o indirectamente en las decisiones del docente sobre la actuación pedagógica y sobre las actividades de lectura y escritura en relación con las representaciones que posee cada agente educativo.

En cuanto al levantamiento de las representaciones discursivas, se utilizan categorías macro pertinentes para la construcción discursiva de la lectura y la escritura: objetivos de lectura y escritura, espacio/tiempo de leer y escribir, modos de leer y escribir, tipos de textos. Dichas unidades se cristalizan de manera diferente en los discursos analizados según el género discursivo al 
que pertenecen. De hecho, la cuestión de la materialidad discursiva de las representaciones en estudio es una cuestión intrincada, pues muchas veces son consideradas implícitamente, por lo que hay que operar con marcas lingüísticas como indicios de procesos no transparentes (Peytard \& Moirand, 1992; Narvaja, 2006). Cabe destacar que estas categorías que permiten el análisis de un corpus heterogéneo, emergieron tanto del trabajo de campo (etnografía educativa interpretativa) como de la revisión bibliográfica (marco de referencia). La perspectiva histórica de los estudios sobre lectura y escritura permitió operacionalizar distintas dimensiones articuladoras de la representación discursiva de la lectura y la escritura. Ahora bien, estas categorías, por su carácter transversal, son definidas para el trabajo de análisis del discurso como unidades no tópicas (Maingueneau, 2005) y fueron construidas con el fin de rastrear en los subcorpus marcas lingüísticas y discursivas que permitan la descripción e interpretación de las representaciones discursivas de los diferentes agentes educativos. Estas unidades no tópicas se vinculan estrechamente con las nociones centrales del modelo dialógico: intertextualidad e interaccionalidad.

Una vez establecidas las categorías, queda aún por establecer la estructura que permite el contraste entre los diferentes discursos y que articula las categorías antes señaladas. Como la lectura y la escritura son acciones, se indaga en el modo en que se escenifica dicha acción en los varios universos discursivos que recrean los distintos discursos que constituyen el corpus de análisis. Para ello, se tomó la estructura actancial que utiliza Moirand (2006a, 2006b) en sus estudios sobre la explicación en los discursos de divulgación científica y se ha trasladado, para este estudio, al campo educativo, pues se considera que dicha estructura permite poner en contacto los aspectos que configuran la representación de la lectura y la escritura:

$$
\begin{aligned}
& \text { A lee [algo] para [algo] } \\
& \text { A escribe [algo] a B para [algo] }
\end{aligned}
$$

Dentro del sistema de enseñanza-aprendizaje, dicha estructura actancial se complejiza, pues los diferentes agentes educativos (administración pública, proyecto educativo del establecimiento en estudio, proyecto familiar, proyecto del docente) pueden tener objetivos de enseñanza para la lectura y la escritura no necesariamente congruentes entre ellos.

\section{A hace que B lea/escriba [algo] para [algo]}

Por lo que se refiere al subcorpus 1 centrado en la actuación pedagógica del docente, dicha estructura se materializa de la siguiente manera: 
$\mathrm{P}($ rofesor) proyecta que $\mathrm{E}$ (studiantes) lean/escriban $\mathrm{T}($ exto) [estructura+género] con tal O(bjetivo) planificación

$\mathrm{P}$ (rofesor) hace que E(studiantes) lean/escriban T(exto) [estructura+género] con tal O(bjetivo) interacción pedagógica

$\mathrm{P}($ rofesor) evalúa E(studiantes) leyendo/escribiendo T(exto) [estructura+género] con tal O(bjetivo) evaluaciones/pruebas

$\mathrm{P}$ (rofesor) dice que hace que $\mathrm{E}$ (studiantes) lean/escriban $\mathrm{T}$ (exto) [estructura+género] con tal O(bjetivo) entrevistas a docentes

Figura 1. Estructura actancial compleja subcorpus 1.

En cuanto al análisis del subcorpus 2, la estructura actancial se despliega de la siguiente manera:

A(dministración pública) proyecta que el $\mathrm{P}$ (rofesor) haga que E(studiantes) lean/escriban T(exto) con tal O(bjetivo)

programa de estudio para los distintos sectores de aprendizaje

$\mathrm{D}$ (irectivos) proyectan que el $\mathrm{P}$ (rofesor) haga que $\mathrm{E}$ (studiantes) lean/escriban $\mathrm{T}$ (exto) con tal O(bjetivo) entrevistas a los directivos y datos contextuales aportados por la etnografía

$\mathrm{F}$ (amilia) proyecta que el $\mathrm{P}($ rofesor) haga que $\mathrm{E}$ (studiantes) lean/escriban $\mathrm{T}$ (exto) con tal O(bjetivo) entrevista a los estudiantes

Figura 2. Estructura actancial compleja subcorpus 2.

Por lo tanto, la descripción e interpretación de las representaciones discursivas sobre la lectura y la escritura se realizan desde el análisis del discurso, lo que implica, necesariamente, la articulación interdisciplinaria de saberes provenientes de distintos campos de la ciencias sociales (educación y antropología) y de las ciencias del lenguaje, pues se busca articular lo social con lo discursivo dentro de una esfera social.

\section{Resultados}

A continuación se presentan los resultados obtenidos del análisis que configuran el corpus de trabajo. 


\subsection{Subcorpus 1: el texto escolar en el centro de la actuación pedagógica}

Con relación a las representaciones discursivas del quehacer pedagógico de los docentes de las cuatro áreas curriculares en estudio, la interpretación se centra en la actuación pedagógica de los docentes (tercer nivel de concreción curricular) para lo que se han considerado los procesos involucrados en la enseñanza-aprendizaje: planificar, actuar/interactuar y evaluar lo que se materializa en una serie de discursos auténticos que configuran estas acciones llevadas a cabo por el docente como agente responsable de la instrucción y enseñanza.

La siguiente figura muestra la manera en que se relacionan los discursos auténticos analizados en el subcorpus 1 .

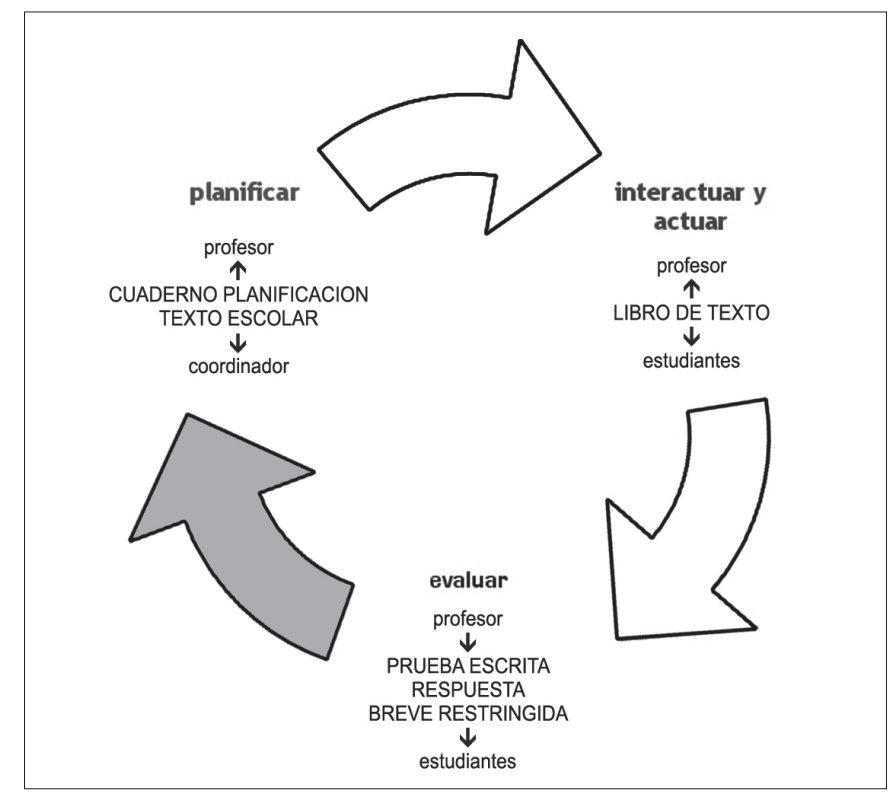

Figura 3. La actuación pedagógica de los docentes de las áreas prioritarias del currículo escolar en NB3 en los discursos auténticos que configuran dicha actuación.

Como muestra la Figura 3, los docentes planifican su actuación en el aula a partir de lo estipulado en los programas de estudio del Ministerio de Educación chileno, guiándose, totalmente, por lo propuesto por los textos escolares licitados por el MINEDUC, lo que se desprende del análisis de lo planificado y como se confirma por voz de los propios docentes en el análisis de lo declarado. La planificación se caracteriza por ser un guión de acciones y actividades por realizar 
durante la clase; no obstante no hay un diseño de unidades didácticas con objetivos y evaluaciones, componentes fundamentales para orientar el proceso educativo. Por otra parte, las actividades diseñadas son enunciadas en las planificaciones mediante la designación de páginas del texto escolar en la que se encuentran consignas de actividades por realizar. Por lo tanto, de las planificaciones emerge una imagen del docente como administrador y ejecutor de actividades diseñadas por otros profesionales externos a la comunidad escolar en estudio.

Asimismo, llama la atención cómo la propuesta de actividades planificadas por los docentes es evaluada por un coordinador, cuyo principal objetivo es supervisar y corregir lo programado por el docente, lo que da cuenta de una propuesta educativa compartida por el segundo nivel de concreción curricular. De lo anterior, se desprende una ausencia de una auténtica programación del aula, ya que el docente no utiliza el texto escolar como un material curricular entre otros para la planificación de sus clases, sino que sigue y ejecuta lo propuesto por el texto escolar, quedando, de este modo, en un papel no protagónico en la secuenciación y organización reflexiva de la acción áulica.

Por su parte, la interacción pedagógica (análisis de lo realizado) se observa fuertemente mediada por el texto escolar en cuanto instrumento pedagógico que presenta tanto los contenidos como las actividades por desarrollar durante la hora de clases. Las intervenciones de los docentes se organizan más bien en torno, tanto a la regulación del comportamiento como a la gestión de actividades, disminuyendo el hilo discursivo de la enseñanza-aprendizaje.

Finalmente, el proceso de evaluación (análisis de lo evaluado) es concebido como calificación de los resultados y no de los procesos de enseñanza-aprendizaje. No se busca, entonces, la información necesaria para contrastar con los objetivos establecidos y los resultados esperados y, así, poder determinar los pasos a seguir tanto para el docente (procesos de enseñanza-instrucción) como para los estudiantes (procesos de aprendizaje) en la búsqueda de la educación. La calificación de los productos es concebida y llevada a cabo al final de un proceso para medir los logros de los estudiantes; en cambio, la evaluación es un proceso permanente y en constante interacción con los otros procesos de enseñanza-aprendizaje.

En cuanto a la lectura y la escritura, se puede concluir que aparece transversalmente en todos los discursos que configuran la actuación pedagógica del docente. Sin embargo, no es representada como un objeto de enseñanza ni siquiera muy claramente en el área de lenguaje. Es más, se utiliza como un medio de acceso y registro de información en todas las áreas curriculares en estudio.

En la cultura escolar, se encontró una conciencia más explícita de la relevancia de la lectura que de la escritura por cuanto existe un programa de lectura elaborado por la dirección y la coordinación del área de lenguaje del establecimiento educacional. Sin embargo, en relación 
con los procesos de enseñanza-aprendizaje, la lectura es utilizada para acceder a los contenidos conceptuales y a las actividades presentes en el texto escolar. La escritura, por su parte, es concebida como un medio de registro de la información necesaria para responder a las actividades del texto escolar.

Con todo, en las clases de las áreas prioritarias del currículo escolar observadas y analizadas emergen en el discurso de los docentes, constantes comentarios e indicaciones sobre el conocimiento grafémico, grafofonémico y ortográfico, principalmente en actividades de copiado y dictado de contenidos conceptuales. Esto -vinculado a la importancia de la lectura de obras literarias- corresponde a una representación de la educación ligada al patrimonio ilustrado, en que el ser humano culto es aquel que posee cierta cantidad de conocimientos legitimados que lo conducirían al prestigio y al ascenso social. Es lo que se ha denominado como "escuela tradicional". Esta visión convive con una propuesta educativa orientada al desarrollo de competencias, núcleo de la propuesta elaborada por la política educativa actual, pero que se presenta como una selección de nuevos contenidos y eliminación de otros más que como un método de enseñanza o una secuenciación didáctica diferente.

En cuanto a las evaluaciones, la lectura y la escritura presentan una función registrativa, ya que son utilizadas por parte del estudiante para responder pruebas escritas (instrumento de evaluación más utilizado) de preguntas cerradas y abiertas de tipo restringido, lo que no permite el uso de la lectura y escritura como una herramienta epistémica.

Si se integra, entonces, lo surgido del análisis de lo declarado por los docentes en las entrevistas (discursos de situación de investigación), se obtiene que el texto escolar se encuentra al centro del proceso educativo, quedando, de esta manera, precisamente ausentes los participantes de este tipo de interacción: profesor y estudiantes.

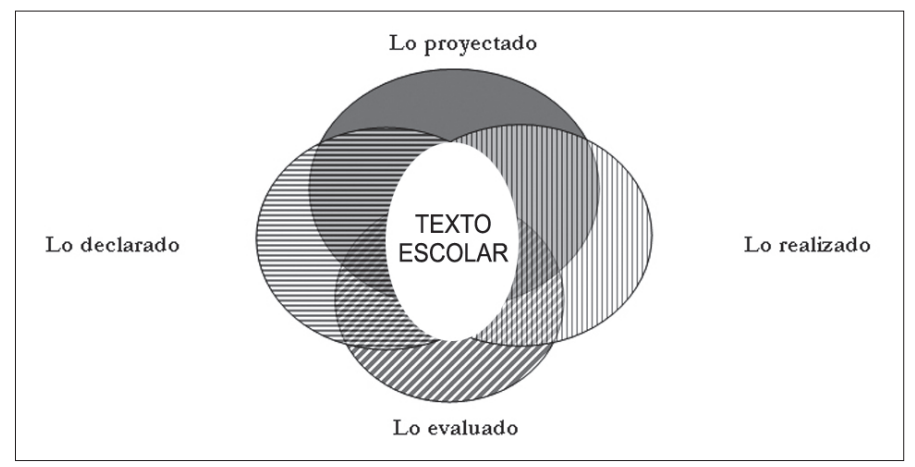

Figura 4. El texto escolar como el material curricular utilizado en el proceso de enseñanza/aprendizaje. 
Del análisis de lo planificado se desprende, por tanto, que hay una ausencia de programación por parte del docente, ya que este enuncia las actividades del texto escolar; lo que es confirmado por el análisis de lo realizado, pues se observa que, en todas las clases del subcorpus 1 , se trabaja con el texto escolar tanto para acceder a los contenidos como para realizar las actividades. Por otra parte, el texto escolar es utilizado como la única fuente de información y estudio puesto que incluso los docentes elaboran un instructivo indicando las páginas del texto escolar que serán evaluadas mediante pruebas de respuesta cerrada (análisis de lo evaluado). En efecto, los propios docentes enuncian (análisis de lo declarado) el uso del texto escolar como principal y casi única herramienta para el desarrollo del proceso de enseñanza-aprendizaje, lo que es explicado como una medida institucional por los bajos resultados obtenidos por el establecimiento educacional en las pruebas nacionales SIMCE, instrumento de medición de la calidad educacional. Según lo comentado por los docentes, es la dirección del establecimiento la que determina la importancia de la cobertura de todos los contenidos del texto escolar como una estrategia para mejorar los puntajes en dicha prueba.

Por lo que se refiere al contraste entre áreas curriculares, dos cosas deben ser destacadas: la primera dice relación con la homogeneidad de las actividades educativas programadas, realizadas y evaluadas debido al uso ya descrito del texto escolar. En segundo lugar, las distinciones entre las distintas áreas no emergen de la propuesta del docente sino más bien de las actividades diseñadas en el texto escolar que enfatizan distintos tipos de procedimientos en los cuales se utiliza la lectura y la escritura. El área Sociedad se caracteriza por tareas de adquisición y análisis de información y la incorporación del trabajo con información gráfica (mapas); Naturaleza, por su parte, presenta tareas didácticas en que se utiliza la lectura y la escritura para el análisis de la información, principalmente en situaciones de experimentación; en Matemática, las tareas poseen objetivos de interpretación de la información, las que se caracterizan por la traducción de un código verbal a un código numérico y, finalmente, en Lenguaje se encuentran sobre todo tareas de análisis y comunicación de información.

En cuanto a la lectura y la escritura, estas aparecen como actividades a realizar en el aula entendida como actividades que median otras acciones pedagógicas; sin embargo, los objetivos de las actividades son bastantes simples desde el punto de vista cognitivo, lo que lleva a concluir que se trata de prácticas de reproducción de la información que, implícitamente, tienen objetivos que se condicen con una visión ilustrada de la educación.

En conclusión, cabe destacar el fuerte componente monológico de esta actuación pedagógica, ya que el docente ejecuta lo determinado por agentes externos a la comunidad educativa sin realizar una actualización y reflexión pertinente para cada grupo-curso. Asimismo, utiliza casi exclusivamente un material curricular, lo que aumenta la primacía de una sola voz en la enseñanza lo que lleva, a su vez, más bien a la reproducción del conocimiento. 


\subsection{Subcorpus 2: el SIMCE y sus efectos retroactivos en el quehacer educativo}

En este apartado se presenta, en primer lugar, el contraste de las representaciones discursivas de la lectura y escritura en relación con los procesos de enseñanza-aprendizaje emergidos del análisis de los discursos de los agentes educativos correspondientes a los dos primeros niveles de concreción curricular y al núcleo familiar.

Con respecto al discurso de la Administración Pública (primer nivel de concreción curricular), resulta interesante destacar que los programas de estudio son configurados en cuanto diseño curricular abierto, ya que solo prescriben orientaciones pedagógicas sobre objetivos, contenidos, actividades y evaluaciones y enuncian los contenidos mínimos y los objetivos fundamentales para cada sector de aprendizaje. Cabe destacar la vastedad y amplitud de los contenidos designados como "mínimos" para cada área curricular. Lamentablemente, una vez realizado el análisis, no se advierte cuál es la hipótesis de significado capaz de integrar todos estos conceptos, cuestión que se agudiza si se observa que estos corresponden a aprendizajes esperados para estudiantes de diez años. Además, en los programas de estudios, se construye la relevancia de la selección de los contenidos (conceptuales y procedimentales) mediante una estrategia de vinculación con la funcionalidad y aplicabilidad de los mismos a las situaciones cotidianas. Esto lleva a reflexionar acerca de la manera en que la política educativa orienta su propuesta en torno a un valor futuro de la educación y no centrado en el valor del presente necesario para cualquier experiencia educativa. En referencia a la representación de evaluación, los programas de estudios construyen una concepción basada en un proceso permanente de entrega de información sobre los procesos educativos en pos de la calidad educativa. Aunque la lectura y la escritura no son mencionadas en las áreas prioritarias, excepto en Lenguaje, como un contenido procedimental que se ha de enseñar, estas aparecen implicadas en la enunciación de las habilidades por desarrollar en cada sector de aprendizaje. Ello revela una concepción subyacente que diferencia entre modos de razonar y el aprendizaje del lenguaje tanto oral como escrito como si, en última instancia, se pudiera razonar sin el lenguaje, sin considerar el aspecto cognitivo y representacional de este y relevando solo su aspecto comunicativo. Esto quiere decir que el ser humano primero razonaría y luego traspasaría esas ideas y las comunicaría a través del lenguaje, sustrayendo, de este modo, toda la dimensión epistémica del lenguaje y, por ende, de la lectura y escritura para la construcción del conocimiento.

En el discurso de los directivos (segundo nivel de concreción curricular), se encontraron marcas sobre los complejos cambios que sufre el establecimiento educacional a raíz del contexto de reforma educativa. Esto quiere decir que la cultura escolar, por condicionamientos del contexto social y político, en estos últimos diez años, ha sufrido, además de los cambios internos anteriormente descritos, importantes cambios propiciados, principalmente, por el discurso nacional acerca de la calidad de la educación y, en particular, por la aplicación de la prueba de medición 
del sistema educativo (SIMCE). En efecto, emerge en el discurso de los directivos la relevancia que ha cobrado dicha prueba como un mecanismo de iniciativa y de control externo. Debido a los bajos resultados obtenidos, hasta el momento se ha generado un control y diseño de propuestas por parte de la dirección del establecimiento para revertir esta situación, que muestra que el núcleo más duro de transformación no es el aspecto estructural de la institución sino el de los saberes compartidos por los agentes que configuran esta cultura escolar. Todo esto ligado a la cuestión económica del SIMCE, cuyos resultados son utilizados como criterio para la asignación de recursos de subvención estatal (Larrañaga, 2004) y como criterio de selección de establecimiento educacional por parte de los padres.

Por lo que se refiere a la lectura y la escritura, en el discurso de los directivos emerge la importancia de la lectura en cuanto parte de una educación ilustrada; sin embargo, no se establecen criterios de evaluación ni objetivos precisos para la construcción de situaciones de enseñanzaaprendizaje en que se advierta la relevancia que posee el lenguaje escrito en el aprendizaje de los contenidos de cada área curricular y en la construcción del conocimiento. Es más, persiste una representación de la lectura vinculada a la literatura y al conocimiento de un patrimonio ilustrado y de la escritura como conservación de la lengua.

Finalmente, el discurso de los estudiantes revela una situación grave en relación con el núcleo familiar: ausencia de adultos que acompañen a los estudiantes en su proceso educativo; se puede advertir que la familia se interesa sobre todo por controlar los resultados obtenidos en el proceso de instrucción escolar y por proveer los recursos económicos y materiales para que estos se logren. Por su parte, los estudiantes se sienten presionados por el rendimiento académico (evaluación como calificación, orientada a los productos) lo que genera en ellos angustia y ansiedad. Por ello, gran cantidad de estudiantes son derivados al experto por problemas conductuales y emocionales. Esto se refuerza con la preferencia que los estudiantes manifiestan por las áreas no prioritarias del currículo escolar como espacio de participación, interacción, expresión y encuentro menos inflexibles.

En cuanto a la lectura y la escritura, los estudiantes la conciben, principalmente en relación con el conocimiento notacional de lo escrito: la lectura como sonorización de letras y la escritura como caligrafía. En ocasiones, varios mencionan como característica relevante de un buen lector la cantidad de obras que lee y de un buen escritor la creatividad, lo que lleva a una concepción de tipo literario de dichas habilidades.

\subsection{Interpretación macro del análisis del subcorpus 1 y del subcorpus 2}

A continuación se presenta, mediante la siguiente figura, el modo en que se relacionan los diferentes agentes educativos en esta cultura escolar en estudio y el tipo de representaciones 
discursivas sobre la lectura y la escritura en relación con los procesos de enseñanza-aprendizaje (teoría sustantiva).

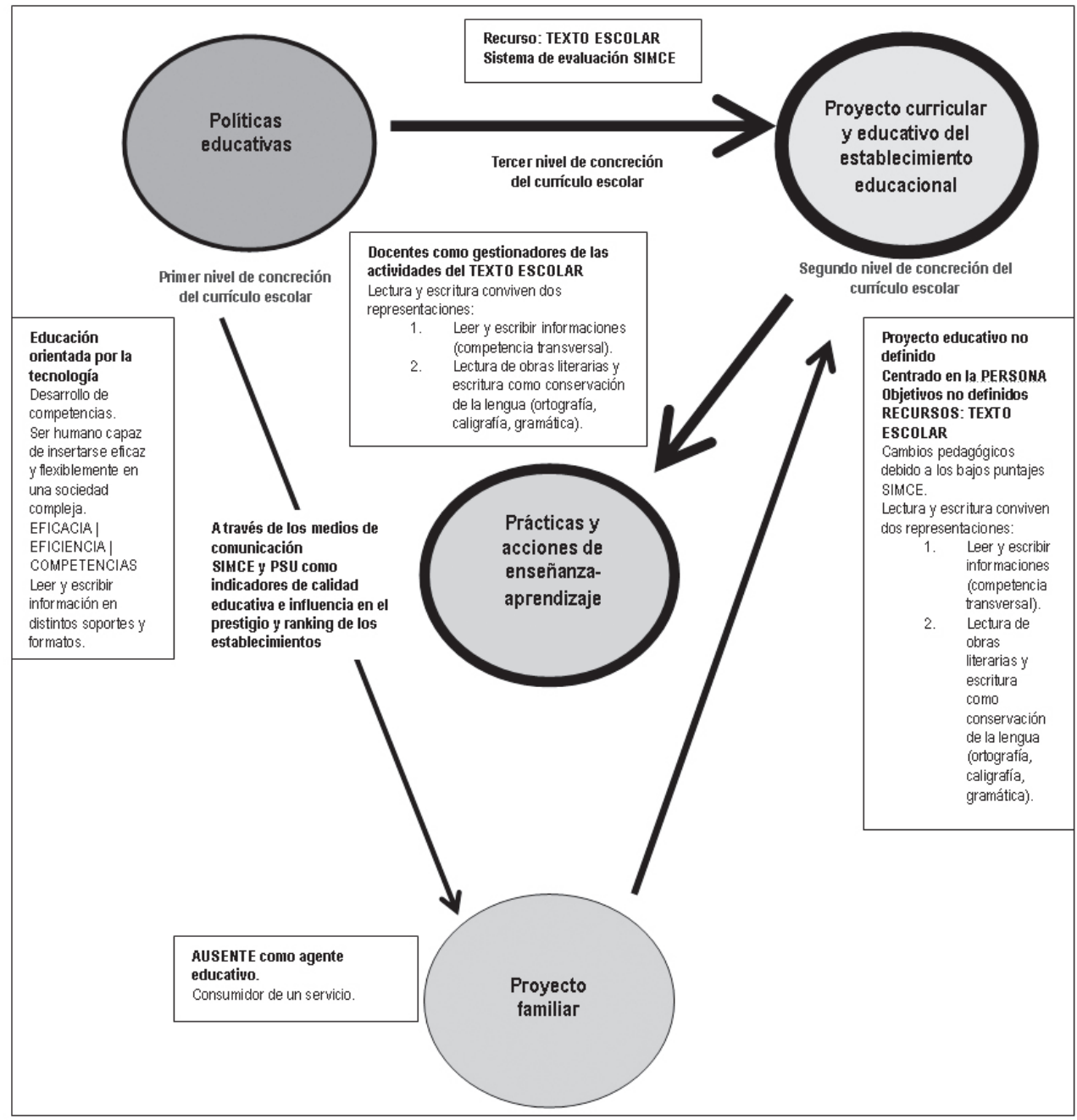

Figura 5. Articulación de las representaciones de la lectura y la escritura en el sistema educativo en los discursos de los distintos agentes educativos que configuran una realidad educativa. 
Como se observa en la Figura 5, se comprueba cómo la política educativa se constituye como el agente más influyente en la programación curricular tanto del centro educativo como de la programación y acción en el aula. Esto se debe, sobre todo, a los efectos retroactivos de la prueba SIMCE sobre todo el sistema: esto quiere decir que el diseño curricular abierto que propone el MINEDUC (análisis de los programas de estudio) es concretizado mediante la elaboración de textos escolares que materializan la propuesta educativa diseñada por la política educativa. Sin embargo, es en el segundo nivel de concreción curricular en el que se comprende el porqué de esta influencia: los establecimientos educacionales son calificados mediante dichas pruebas según parámetros leídos por la comunidad escolar y por los medios de comunicación como un criterio de clasificación de los establecimientos educacionales. Por ende, obtener bajo puntaje en el SIMCE es percibido como un indicador de mala calidad de la educación impartida por el centro. De ahí, entonces, que la dirección del establecimiento genere mecanismos de control y de diseño curricular orientados a la mejora en dichas pruebas, las que miden más bien resultados y no el proceso educativo. Por lo tanto, se implementan una serie de medidas, generadas desde la dirección y no desde la comunidad, para revertir esta situación: planificación y realización de todo lo estipulado en los textos escolares del MINEDUC.

De esta manera, en el tercer nivel de concreción curricular -el más relevante del proceso educativo- el docente ejecuta lo fijado en el texto escolar dando prioridad a la cobertura total del programa de estudio, concretizado en el libro de texto, y no a los aprendizajes esperados. Ahora bien, esto lleva a reflexionar acerca de la determinación de objetivos externos por parte del establecimiento educacional, lo que se corresponde con la falta de un proyecto curricular propio.

Por último, el núcleo familiar se ha desligado, por un sinnúmero de razones, de la educación de sus hijos, confiando dicha tarea al sistema educativo. Los padres esperan que el establecimiento educacional otorgue lo necesario para asegurar el éxito y la inserción de sus hijos en la competitiva sociedad actual. Como emerge del discurso de los estudiantes, están orientados hacia el futuro y hacia los resultados, lo que distorsiona el proceso educativo.

En cuanto a la lectura y la escritura, es concebida en estrecha relación con el texto escolar como medio de acceso y manejo de información; sin embargo, no se enuncia como un objeto de enseñanza vinculado con el aprendizaje en los distintos sectores del currículo escolar. Asimismo, como la escuela establece como prioridad la cobertura de los contenidos -los cuales son máximos y no mínimos- la lectura y la escritura no se conciben como contenidos procedimentales, sino que se utilizan solamente como un medio. En efecto, persiste más bien la representación ligada al conocimiento notacional, objeto de enseñanza del primer ciclo educativo. Por ende, se asume que una vez que los estudiantes aprenden a codificar y decodificar ya han aprendido la lectura y escritura y después la utilizan, pero no se introduce a los estudiantes en el lenguaje 
escrito y en su relación con el aprendizaje y la construcción del conocimiento (herramienta epistémica). Por otra parte, se releva en los discursos una representación de la cultura escrita ligada al pensamiento ilustrado y a la conservación de la lengua como un saber prestigioso, pero que, en última instancia, se concibe como un espacio desvinculado de las áreas prioritarias del currículo escolar. Dicho programa, al no presentar objetivos ni mecanismos de evaluación adecuados, es percibido por la comunidad como prácticas sin sentido para el aprendizaje. Por lo tanto, de este análisis se desprende la importancia del segundo nivel de concreción curricular, como espacio vital de cambios significativos en la educación, ya que estos no se establecen solo por cambios en la estructura social, sino ante todo en el proyecto curricular a través del cual se establecen objetivos claros vinculados con una concepción de educación y del ser humano que se desea formar. De este segundo nivel es desde donde puede generarse una comunidad escolar compuesta por seres humanos que trabaja colaborativamente en pos de la educación.

\section{CONCLUSIONES}

Del análisis de los distintos discursos que circulan y configuran la cultura escolar como espacio comunicativo, se concluye, en primer lugar, que conviven por lo menos, dos tipos de representaciones al considerar la dimensión cognitiva/representacional de los discursos analizados (dialogismo intertextual que da cuenta de la construcción del objeto representado en los distintos discursos analizados):

1) Leer y escribir para manejar información y generar conocimiento, representación propia de una visión de la educación orientada por la tecnología. Dicho modelo educativo promueve el desarrollo de la lectura y escritura en cuanto competencia transversal del currículo escolar y ligado principalmente al concepto de información, promoviendo el sistema educativo aprendizajes que permiten un trabajo eficiente y eficaz con diversos tipos de informaciones (gráfica, numérica y verbal).

2) Leer literatura y escribir para aprender el correcto uso de los aspectos notacionales de la lengua escrita (ortografía, vocabulario y caligrafía) representación propia de una visión de la educación orientada al patrimonio ilustrado. Dicha propuesta educativa busca la formación de un intelectual capaz de reflexionar y de poseer una serie de conocimientos considerados fundamentales para el ejercicio de la ciudadanía. Sin embargo, claramente, esta representación ha perdido su ligamen con los objetivos propios de la educación ilustrada y ha permanecido como una representación persistente de un tipo de educación calificada como "tradicional".

Concordantemente, de la observación de lo realizado en clase, se evidencia que, al interior del aula, la lectura y escritura se utilizan, principalmente, como un instrumento de reproducción 
de información: copiado y acceso. Esto pone de manifiesto la tensión existente entre las representaciones descritas anteriormente, en cuanto ancladas a modelos pedagógicos diferentes, y correspondería a una asimilación acrítica dentro de un complejo proceso de transición relacionado con la reforma educativa.

En segundo lugar, al considerar la dimensión comunicativa propia de todos discursos analizados (dialogismo interaccional en cuanto lo representado responde a una selección estratégica de lo enunciado frente a un destinatario o sobredestinatario), se puede concluir que dichas representaciones "simbióticas" son interpretadas dentro del sistema educativo como parte de los efectos retroactivos sobre los procesos de enseñanza-aprendizaje de las pruebas estandarizadas que se aplican actualmente en los establecimientos educacionales chilenos, para medir la calidad educacional. Esto quiere decir, que todo el proceso educativo en cuanto diseñado por los directivos y los docentes, y realizado por estos últimos, está orientado y dirigido para responder a lo evaluado y calificado por la prueba estandarizada para medir la calidad de la educación. De esta manera, gran parte de las decisiones -como ponen en evidencia los indicios lingüísticos-discursivos relevados en el análisis del discurso de los distintos agentes del sistema educativo- están en diálogo con los criterios de calidad estipulados por la administración pública en cuanto sobredestinatario, entendido como el grupo social que determina lo considerado "valioso" en términos educativos.

Desde el macrocontexto, se advierte que la escuela en la que se ha llevado a cabo el estudio se inserta en el escenario de la "sociedad de la información", cuya característica principal es la de otorgar al ser humano toda la información que quiera -cuestión observada en el diseño abierto del currículo escolar- sin otorgarle un punto de interpretación que le confiera sentido a todo lo recopilado. Esto es explicado desde el modelo educativo nihilista que renuncia a una propuesta educativa orientada por el sentido (Morandé, 2003) y, a cambio, presenta programas de estudio de una amplia cobertura temática declarando, ante todo, el desarrollo de competencias transversales para "aprender a aprender"; por lo mismo, la escuela en cuanto proyecto curricular debe plantearse la necesidad de proponer un punto de observación, una hipótesis explicativa que logre interrelacionar todos estos datos, que logre mostrar el sentido de estos conocimientos y que utilice para ello la lectura y la escritura como herramienta para la construcción del conocimiento y la formación de la autoconciencia del ser humano. Asimismo, se advierte cada vez más la separación entre la escuela y la experiencia. El problema de la educación no radica tanto en la inserción en el mundo laboral y profesional (educación orientada a lo futuro y a los resultados, búsqueda del éxito) sino en los modos en que se presenta a los jóvenes la tradición cultural que se transmite tanto en los textos como en la experiencia del docente (educación en el presente para el descubrimiento de las causas originales y el sentido de las cosas). 


\section{REFERENCIAS BIBLIOGRÁFICAS}

Adam, J. (1999). Linquistique textuelle. Des genres de discours aux textes. Paris: Nathan.

Adam, J. (2005). La linguistique textuelle. Introduction à l'analyse textuelle des discours. Paris: Armand Colin.

Cambra, M. (2003). Une approche ethnographique de la classe de langue. Paris: Didier.

Charaudeau, P. (2000). Las problemáticas de base de una lingüística de discurso. En J. De Bustos, P. Charaudeau, J. Girón, S. Iglesias \& C. Lopez (Eds.), Lengua, discurso, texto (I simposio internacional de Análisis del Discurso) (pp. 39-52). Madrid: Visor.

Charaudeau, P. \& Maingueneau, D. (Eds.) (2005). Diccionario de análisis del discurso. Buenos Aires: Amorrortu.

Grize, J. (1996). Logique naturelle et communications. Paris: Presses Universitaires de France.

Larrañaga, O. (2004). Competencia y participación privada: La experiencia chilena en educación. Santiago de Chile: Universidad de Chile.

Maingueneau, D. (2003). Términos clave del análisis del discurso. Buenos Aires: Nueva Visión.

Maingueneau, D. (2005). L'analyse du discours et ses frontières. Marges linguistiques, 9, 64-75.

MINEDUC (2004a). Desempeños a lograr en Lectura, Escritura y Matemática (2NT, NB1, NB2) [en línea]. Disponible en www.mineduc.cl

MINEDUC (2004b). Prueba SIMCE $2^{\circ}$ Medio 2003. Análisis de Resultados. MINEDUC [en línea]. Disponible en: www.mineduc.cl

Moirand, S. (2001). Du traitement différent de l'intertexte selon les genres convoqués dans les événements scientifiques à caractère politique. Semen, 13, 97-117.

Moirand, S. (2003a). Communicative and cognitive dimensions of discourse on science in the French mass media. Discourse Studies, 5(2), 175-206.

Moirand, S. (2003b). Quelles catégories descriptives pour la mise au jour des genres du discours? [en línea]. Disponible en: http://gric.univlyon2.fr/Equipe1/actes/journees_genre.htm

Moirand, S. (2004a). Le texte et ses contextes. En J. Adam, J. Grize \& A. Boucha (Eds.), Textes et discours: Catégories pour l'analyse (pp. 129-144). Paris: Editions Universitaries de Dijon.

Moirand, S. (2004b). L'impossible clôture des corpus médiatiques. La mise au jour des observables entre catégorisation et contextualisation. Tranel, 40, 71-92.

Moirand, S. (2005). Le dialogisme, entre problématiques énonciatives et théories discursives. Cahiers de praxématique, 43, 189-220.

Moirand, S. (2006a). La divulgación de la ciencia y la técnica: ¿Nuevos modelos para nuevos objetos de estudio? Revista Signos. Estudios de Lingüística, 39(61), 231-358.

Moirand, S. (2006b). Un modèle dialogique de l'explication. En C. Hudelot, A. Salazar-Orvig \& E. Ve- 
neziano (Eds.), L'explication: Enjeux cognitifs et communicationnels (à paraître) (pp. 77-78). Bruxelles: Peeters.

Morandé, P. (2003). Conocimiento, información y sabiduría. Humanitas, 30, 247-260.

Narvaja, E. (2006). Análisis del discurso. Modos de abordar materiales de archivo. Buenos Aires: Santiago Arcos.

Peytard, J. \& Moirand, S. (1992). Discours et enseignement du francais. Paris: Collection Références. 